\title{
Forced Swimming and Crocin Supplementation Protects Rat Cardiomyocytes From H2O2囚Induced Apoptosis
}

\section{Mina Akbari}

Shahid Rajaee Teacher Training University

Fereshteh Shahidi ( $\square$ Angel.shahidi@yahoo.com )

Shahid Rajaee Teacher Training University https://orcid.org/0000-0001-6500-289X

Hamid Rajabi

Tehran University of Teacher Training: Kharazmi University

Majid Kashef

Shahid Rajaee Teacher Training University

\section{Research Article}

Keywords: Forced swimming, Crocin supplement, BAX, BCL-2, H2O2, Caspase3

Posted Date: February 13th, 2021

DOl: https://doi.org/10.21203/rs.3.rs-186119/v1

License: (c) (i) This work is licensed under a Creative Commons Attribution 4.0 International License.

Read Full License 


\title{
Forced swimming and Crocin supplementation protects rat cardiomyocytes from $\mathrm{H2O2}$ - induced apoptosis
}

\author{
Mina Akbari ${ }^{1}$, Fereshteh Shahidi $^{*}$, Hamid Rajabi ${ }^{3}$, Majid Kashef ${ }^{4}$ \\ ${ }^{1}$ Faculty Exercise Physiology at Shabid Rajaee Teacher Training University, Tebran, Iran \\ ${ }^{2}$ Faculty of Exercise Physiology at Shabid Rajaee Teacher Training University, Tehran, Iran \\ 3Department of animal physiology, Faculty of Biology, Kharazmi University, Tehran, Iran \\ ${ }^{4}$ Professor of Exercise Physiology at Shahid Rajaee Teacher Training University, Tehran, Iran
}

* Corresponding author: Department of Physiology, Faculty of Physical Education and Sport Sciences, Shahid Rajaee Teacher Training University, Tehran, Iran. Tel.: +989122970414; fax: +982122970060.

E-mail address: shahidif2020@gmail.com (F. Shahidi).

\section{Co-author contact information:}

Mina Akbari - email: (akbariteach@gmail.com)

Hamid Rajabi - email: (hrajabi1346@yahoo.com)

Majid Kashef - email: (kashef1337@gmail.com) 


\begin{abstract}
Introduction: Oxidative stress and hypoxia/reperfusion can damage cells and lead to apoptosis in cardiomyocytes. Physical activities and antioxidants supplementations have shown to have potent antiapoptotic properties. This study aimed to determine the interactive effect of forced swimming and Crocin supplementation on apoptotic and anti-apoptotic cardiomyocytes gene expression in male rats infected with $\mathrm{H} 2 \mathrm{O} 2$.
\end{abstract}

Methods: Thirty-six male Wistar rats were divided randomly into six groups of sham, control, H2O2, $\mathrm{H} 2 \mathrm{O} 2+$ forced swimming, $\mathrm{H} 2 \mathrm{O} 2+$ Crocin, $\mathrm{H} 2 \mathrm{O} 2+$ forced swimming, and $\mathrm{H} 2 \mathrm{O} 2+$ forced swimming + Crocin. The forced swimming protocol was two stages. Crocin injected a dose of 12.5 $\mathrm{mg} / \mathrm{kg}$ through the intraperitoneal route. Real-time PCR was used to reveal the expression of Bcl2, Bax, and Caspas3 genes. Also, apoptosis was evaluated by TUNEL staining.

Results: H2O2 exposure was associated with a significant increase in the apoptotic gene expression of BAX and caspase3 and a decrease in anti-apoptotic BCL-2 gene expression. Administration of Crocin and six weeks of forced swimming resulted in a significant decrease in the expression of BAX. Also, treatment accompanied by forced swimming and Crocin significantly reduced the expression of caspase 3 and increased BCL-2 expression as compared to the $\mathrm{H} 2 \mathrm{O} 2$ group.

Conclusion: Our results reveal that regular forced swimming and Crocin consumption led to a significant reduction in gene expression of BAX and caspase 3 and the significant increase in antiapoptotic gene expression of BCL-2 in rats' cardiomyocytes.

Keywords: Forced swimming; Crocin supplement; BAX; BCL-2; H2O2; Caspase3

Key point: Saffron is the best herbal supplement to prevent tissue damage. Swimming has many health benefits. 


\section{Introduction}

According to the World Health Organization (WHO), myocardial ischemia is one of the leading causes of death in the world by 2020 . Therefore, cardiac injuries are a very common phenomenon and the development of protective strategies against these disorders is very important (Ascensão, et al. 2007; MOHSENIZADEH, et al. 2017). Several factors contribute to the development of cardiovascular disease (CVDs), The generation of ROS specifically from the myocardium has been implicated in further mitochondrial dysfunction and adjustment of mitochondrial DNA. Harm to mitochondrial DNA leads to impaired synthesis of mitochondrial proteins and raise further ROS generation (Faria and Persaud 2017) Most CVDs are accompanied by an imbalance between the reactive oxygen species (ROS) generation and antioxidant enzymes that subsequently resulted in deviation from the stable state (Aruoma 1998; Ghavami, et al. 2005).

Apoptosis is a type of programmed cell death, and there are two main apoptotic signaling pathways in the cardiovascular system. Also, the intrinsic pathways and extrinsic pathways are associated with ischemic heart diseases [5]. Oxidative stress is established by the loss of balance among the production of free radicals and neutralize the effect of free radicals by antioxidant agents (Granger and Kvietys 2015; Halliwell and Gutteridge 2015), and can cause tissue damage through the peroxidation of lipids, proteins, and the activation of pathways ending apoptosis [5]. Hydrogen peroxide is a mild and relatively stable oxidant, which has widely used as an indicator of ROS to evaluate the cell responses to oxidative stress (Maletic, et al. 1999). There is evidence that oxidative stress induces a destructive effect on cells by disturbing intracellular apoptosis signaling pathways (Kelly 2003). Several studies have shown that ischemia and reperfusion also produce free radicals such as ROS, and gradually cause thrombosis, cell death, and damage to coronary arteries in male rats 
(Persson, et al. 2014). It has been suggested that different ROS production stimulate apoptotic signaling pathways in myocardial cells. In the same vein, the brief exposure of $\mathrm{H} 2 \mathrm{O} 2$ in cardiac cells can stimulate the pathologic mechanisms that cause cell damage, as well as the release of cytochrome C into cytosolic cells of the heart (Lajoie, et al. 2004). Therefore, it is likely that ischemia and reperfusion (I/R), ROS, and also hydrogen peroxide activate the same apoptotic pathway.

The mitochondrial membrane permeability to cytochrome $\mathrm{C}$ has been determined by the relative proportions of pro-apoptotic intermediates such as Bax or Bak, and BCL-2. It is necessary to explain that two apoptosis pathways (internal and external) ultimately activate caspases (Pisoschi and Pop 2015). Caspases as part of the cysteine proteases family play a pivotal role in the onset and different phases of apoptosis, act on specific substrates, and imply morphological changes in the apoptotic cells, including cellular wrinkling and chromatin condensation. Therefore, the activity of caspases is considered as an apoptotic biochemical function (Kelly 2003; Maletic, et al. 1999). On the other hand, the stimulatory effect of exercise on the production of ROS is an important phenomenon of adaptive exercise, which increases resistance to long-term oxidative stress and is a valuable physiological strategy for pre-conditioning myocardial infarction (Plant, et al. 2003; Radák, et al. 2000).

Overall, physical activity has the potential to modulate proliferation and cell death through cytokines, hormones, growth factors, and metabolic pathways. However, long-term endurance training can alter the expression of the gene of BAX and BCL-2 proteins and stimulate cardiac apoptosis by oxidative stress (Ghavami, et al. 2005; Persson, et al. 2014). These data reveal that exercise has a protective effect on the rat's heart. Therefore, exercise seems to be beneficial in some way through reducing ROS generation and preventing the release of intra-mitochondrial cytochrome $\mathrm{C}$ to the reduction of cardiac apoptosis (Roy and Nicholson 2000). Crocin, a natural dietary carotenoid, is the major active constituents of Crocus sativus L (saffron) (Arshadi, et al. 2015). Studies have reported the anti-apoptotic effects, free radicals inhibition, and antioxidant properties of crocin (Sallam and 
Laher 2016). Also, studies have indicated the anti-apoptotic effects of crocin in the cardiovascular system (Pisoschi and Pop 2015). Some researchers believe that physical exercise could decrease apoptosis through the reduction of oxidative stress in the animal heart, Anti-apoptotic effects of crocin in apoptosis induced by increasing the glutathione level, increasing the Bcl2 / Bax ratio, decreasing the release of cytochrome $\mathrm{C}$ into the cytosol, and controlling the activation of Caspase 3 in cardiac tissue (Roy and Nicholson 2000). Overall, studies have shown that physical activity with alteration of apoptotic and pro-apoptotic genes both internal and external pathways resulted in changes in cell apoptosis. Furthermore, researches have shown the beneficial effects of Crocin, including antioxidant properties, free radicals inhibition, and so on. Therefore, this study aimed to investigate the effects of forced swimming and Crocin supplements on apoptotic and anti-apoptotic cardiomyocytes gene expression in male rats infected with $\mathrm{H} 2 \mathrm{O} 2$.

\section{Materials and methods}

Thirty-six male Wistar rats, 6-8 weeks of age weighing 200-250 g, were purchased from the Experimental Animal Center of the Shahid Rajaee Teacher Training University. The animals were allowed free access to food and water and were exposed 12 hours in light and 12 hours in darkness for 21 days in a room, at a temperature of $22-24^{\circ} \mathrm{C}$, with humidity of $40-60 \%$, to adapt to the environment. All procedures involving animals were performed according to the National Institutes of Health Guidelines on the Use of Laboratory Animals (NIH publication No. 85-23, revised1996), and were approved by the Shahid Rajaee Teacher Training University. After one week to adapt to the new environment, animals were randomly divided into six groups: 1) sham injections (saline), 2) $\mathrm{H} 2 \mathrm{O} 2$, 3) $\mathrm{H} 2 \mathrm{O} 2$ + Crocin, 4) $\mathrm{H} 2 \mathrm{O} 2$ + forced swimming, 5) $\mathrm{H} 2 \mathrm{O} 2$ + forced swimming +Crocin, and 6) control. 


\section{Forced swimming protocol}

The forced swimming protocol was held in two stages in training and practice. The training phase included the first week, which on the first day, rats in a standard repository $(140 * 60 * 45 \mathrm{~cm})$ made by Electro company model KAHR0BA20A and 776929906 in the laboratory of Shahid Rajaee Teacher Training University for 10 minutes at a water temperature from 33 to 36 degrees Celsius in turbulent water. The activity period was extended every 10 minutes to allow rats to function in water for 60 minutes. Then, the forced swimming stage was 60 minutes a day and 5 days a week, and in general for 6 weeks. Forced swimming, because it is an intrinsic activity in rats, is also not harmful to the legs and is physically less damaging to the rat (Arshadi, et al. 2015).

\section{Induction of $\mathrm{H} 2 \mathrm{O} 2$}

For the $\mathrm{H} 2 \mathrm{O} 2$ induction groups at $1 \mathrm{mg} / \mathrm{kg} / \mathrm{kg}$ body weight for 3 weeks and one day in the peritoneum on the right leg of the rats and 30 minutes before the exercise, 25 (H2O2 injection was 30\% and was ordered by Merchandise Company by Clean Chemical Company.

\section{Crocin treatment}

Crocin was prepared with $98 \%$ purity from the German company Sigma, manufactured by Kimia Gostar, which was diluted in $2.5 \mathrm{cc}$ doses of $2.5 \mathrm{~g} / \mathrm{kg}$ daily with $5 \mathrm{ccs}$ distilled water and injected with the insulin syringe freshly in the peritoneum. This injection was performed in the combination group for the induction of Crocin and $\mathrm{H} 2 \mathrm{O} 2$ immediately after the injection of hydrogen peroxide and in the left leg of the rats and lasted 3 weeks (Lajoei et al., 2004). Given the fact that $\mathrm{H} 2 \mathrm{O} 2$ studies have shown a dual effect, there is no intervention with Crocin injections (Lajoie, et al. 2004). 


\section{Sampling}

Rats were sacrificed by $\mathrm{CO} 2$ gas 48 hours after the last session of forced swimming and after 10-12 hours of fasting by CO2 gas. After abdominal cavity splitting, carefully separate the heart tissue and after washing were rapidly frozen in liquid nitrogen and stored at $-70{ }^{\circ} \mathrm{C}$.

\section{Apoptosis assay}

RNA extraction, cDNA synthesis, and real-time PCR Quantitative real-time reverse transcription-polymerase chain reaction (RT-qPCR) were performed for determining the expression levels of desired Genes. Total RNA was extracted from samples, by the TRIZOL reagent (Invitrogen; Thermo Fisher Scientific, Inc., Waltham, MA, USA). The purity of RNA samples and integrity were measured with a Nanodrop ND-1000 spectrophotometer; Thermo Fisher Scientific, Waltham, MA) and electrophoresis, respectively. According to manufacturer instructions, cDNA synthesis was done by PrimeScript ${ }^{\mathrm{TM}}$ 1st strand cDNA synthesis kit (TaKaRa, \#6110A, Japan) Briefly, after DNase treatment, total RNA $(2 \mu \mathrm{g})$ was reversed to cDNA by oligodT primers (1 $\mu \mathrm{l}$ of $100 \mu \mathrm{M})$, RiboLockRNase-inhibitor $(0.5 \mu \mathrm{L}$ of $40 \mathrm{U} / \mu \mathrm{L})$, dNTPs $(2 \mu \mathrm{L}$ of $10 \mathrm{mM})$, and MMuLVRevertAid Reverse Transcriptase $(1 \mu \mathrm{L}$ of $200 \mathrm{U} / \mu \mathrm{L})$, incubated for $10 \mathrm{~min}$ at $25^{\circ} \mathrm{C}$, followed by $60 \mathrm{~min}$ at 42 ${ }^{\circ} \mathrm{C}$ in a total volume of $20 \mu \mathrm{L}$. The reaction was terminated using heating the reactions at $70{ }^{\circ} \mathrm{C}$ for 10 min. Primers for real-time PCR were designed with the Oligo7 software, and primer sequences are shown in Table 1. Real-time PCR amplification was performed in a MIC real-time PCR system (BioMolecular systems, London, UK) and the following thermal cycling conditions: Initial denaturation in $95^{\circ} \mathrm{C}$ for $5 \mathrm{~min}, 40$ cycles of $95^{\circ} \mathrm{C}$ for $15 \mathrm{sec}, 57^{\circ} \mathrm{C}$ for $30 \mathrm{sec}$, and $72^{\circ} \mathrm{C}$ for $25 \mathrm{sec}$. The expression of each amplicon was analyzed using the $2-\Delta \Delta$ Ct method (Moradi, et al. 2019). 


\section{TUNEL staining}

The TUNEL staining kit (Roche, Germany) was used to detect apoptosis in cardiomyocyte tissues. Brain tissues were fixed in 4\% formaldehyde ( $\mathrm{pH} 7.4)$ and then embedded in paraffin blocks. Then, serial sections $4 \mathrm{~lm}$ thick were cut and after deparaffinization and rehydrating, stained with TUNEL. The TUNELpositive cells were counted for five fields per section under a light microscope, and data were expressed as the number of TUNEL-positive neurons per $\mathrm{mm} 2$.

\section{Statistical analysis}

The data were analyzed by GraphPad Prism 6 (GraphPad Software, USA), and are represented as Mean \pm standard error of the mean (SEM). Statistical significance was assessed using a one-way analysis of variance (ANOVA), following by a post hoc test (Tukey's method). Differences were considered significant when $\mathrm{p}<0.05$.

\section{Results}

\section{BCL-2 expression}

As shown in Figure 1, the results showed that the induction of $\mathrm{H} 2 \mathrm{O} 2$ resulted in a significant decrease in BCL-2 expression $(\mathrm{P}<0.01)$. After 6 weeks of treatment of rats with crocin and exercise, there was a significant increase in the expression of BCL-2 in the combination treatment group in comparison with the $\mathrm{H} 2 \mathrm{O} 2$ group $(\mathrm{P}<0.001)$.

\section{BAX expression}

As depicted in Figure 2, the induction of $\mathrm{H} 2 \mathrm{O} 2$ significantly increased BAX expression in cardiomyocytes $(\mathrm{p}<0.001)$. On the other hand, all interventions significantly downregulated the expression of BAX compared to the $\mathrm{H} 2 \mathrm{O} 2$ group $(\mathrm{p}<0.001)$. 


\section{Caspase 3 expression}

$\mathrm{H} 2 \mathrm{O} 2$ induction also led to a significant increase in expression of caspase- 3 in comparison to the control group $(\mathrm{P}<0.001)$, while crocin and/or exercise alone and together markedly decreased caspase 3 expression as compared to the $\mathrm{H} 2 \mathrm{O} 2$ group $(\mathrm{P}<0.001)$ (Figure 3).

\section{Effect of crocin and exercise on apoptosis}

Our results showed (Figure 4) that the apoptotic cell number was significantly increased in the cardiomyocytes of the $\mathrm{H} 2 \mathrm{O} 2$ group in comparison to the control. However, the number of apoptotic cells was reduced in the crocin treated group in comparison to the $\mathrm{H} 2 \mathrm{O} 2$ group. Moreover, in crocin together with the exercise group, the number of apoptotic cells was significantly decreased compared to the $\mathrm{H} 2 \mathrm{O} 2$ group.

\section{Discussion}

In the current study, the effects of forced swimming accompanied by Crocin has been investigated in $\mathrm{H} 2 \mathrm{O} 2$ induced poisonous rats to evaluate the expression of apoptotic and antiapoptotic genes in the heart tissue of male Wistar rats. Our findings showed that $\mathrm{H} 2 \mathrm{O} 2$ induction resulted in a significant increase in the apoptotic gene expression of BAX and Caspase 3. Furthermore, six weeks of forced swimming led to a remarkable reduction in the expression of the anti-apoptotic gene of BCL-2 in the myocardial tissues, as well as induction of Crocin as an exogenous antioxidant caused a significant decrease in apoptotic gene expression of BAX, Caspase 3 in myocardial tissues. Also, the interaction between forced swimming and induction of Crocin and oxidation of forced swimming had a decreasing effect on the apoptotic expression of BAX and Caspase 3, and an 
increasing effect on the expression of an anti-apoptotic gene of BCL-2, which indicates the protective effect of regular activity in reducing cardiomyocyte apoptosis. The percentage of apoptosis induced by hydrogen peroxide is $68 \%$, which this amount decreased to $28 \%$ in forced swimming and Crocin intake. Also, forced swimming and crocin intake separately led to a significant reduction in gene expression, apoptotic BAX, caspase 3, and a significant increase in anti-apoptotic gene expression of BCL-2 in rats' myocardium.

Faria et al. have shown that long-term endurance exercises can alter the BAX gene expression and BCL-2 proteins, also stimulate cardiac apoptosis by inducing oxidative stress (Faria and Persaud 2017). However, Sallam et al indicated that exercise training reduces the upstream pro-apoptosis signaling in the BCL-2 family by decreasing caspase 9, Bax, as well as increasing BCL-2, which resulted in a decrease in the Bax / BCL-2 ratio. These data show that exercises have a protective effect on the heart of the rats (Sallam and Laher 2016). Also, Oruc et al. observed that 10-week regular forced swimming in male rats led to an increase in anti-apoptotic proteins (BCL-2, Bcl-x) and a decrease in the induction of proteolytic apoptosis. Therefore, forced swimming seems to be beneficial in some way by reducing ROS generation and preventing the release of intra-mitochondrial cytochrome $\mathrm{C}$ to reducing cardiac apoptosis (Oruc, et al. 2016).

It has been observed that exogenous $\mathrm{H} 2 \mathrm{O} 2$ with a concentration of $2 \mathrm{mmol} / \mathrm{L}, 5 \mathrm{mmol} / \mathrm{L}$, and $1 \mathrm{mmol} / \mathrm{L}$ causes significant changes in energy metabolism in rat reticulocytes (Maletic, et al. 1999). Chronic administration of $\mathrm{H} 2 \mathrm{O} 2$ after exercise induces a reduction in carbonyl groups to below the base level and stimulates the activity of protease and DT-defrazas in the heart muscles (Xiang, et al. 2006). Therefore, the induction of physical exercise due to the production of free radicals is a critical phenomenon in the compromise of sports activity because it increases resistance to oxidative stress. Regular exercise is a good physiological tool for the pre-preparation of myocardium against long-term oxidative stress (Radák, et al. 2000). In another study investigating the effects of long-term aerobic 
activity on apoptosis in normal rats, the measurement of pro-apoptotic and apoptotic proteins showed a decrease in BCL-2 levels compared to the Bax level immediately after aerobic exercise (RADak, et al. 1999). While its ratio was reversed 96 hours after aerobic activity. Therefore, an aerobic activity field increases cell death immediately after aerobic activity, while the survival rate of the cells increases four days later (5). Accordingly, long-term aerobic exercise may have a positive effect on cell survival. Therefore, apoptosis occurs excessively in response to functional demands (Quindry, et al. 2005). Inline, in another study using electrophoretic gel in rat muscle with dystrophin poverty, 48 hours after running on the wheel, DNA destroyed, and BCL-2 levels decreased. The low levels of this antiapoptotic mitochondrial protein may explain the greater involvement of apoptosis following acute activity. Besides, muscular urinary iodine fixation (increased ubiquitin in the muscle nucleus) in both healthy runners and MDX increased in comparison to healthy rats (Zhang, et al. 2020). On the other hand, Crocin inhibits apoptosis in these cells in the presence of end-glucose products in the presence of endothelial cells in the cow's aorta in vitro by increasing the activity of superoxide dismutase and decreasing the concentration of malondialdehyde and oxygen radicals as well as intracellular freecalcium ions in endothelial cells. It has been suggested that crocin may prevent the onset of diabetic vascular complications, such as atherosclerosis (Verma and Bordia 1998). Ghavami et al. showed that by increasing the BCL-2/Bax expression ratio, Crocin could be inhibited apoptosis of endogenous endothelial cells induced by $\mathrm{H} 2 \mathrm{O} 2$-induced bovine aorta. Similarly, another study on Cows aortic endothelial cells showed that Crocin had a preventive effect on the cellular apoptosis induced by H2O2 (Ghavami, et al. 2005). Crocin was significantly inhibited in bovine concentrations of 1-10 $\mu \mathrm{m}$ due to morphological changes induced by apoptosis induced by $\mathrm{H} 2 \mathrm{O} 2$ which, in the form of nucleus congestion, the formation of sprouts on the membrane surface, and the formation of apoptotic bodies, significantly prevailed and in the recipient group Crocin $10 \mu \mathrm{m}$, no DNA laden state. Also, the protection role of Crocin in apoptosis induced by advanced glycation products in endothelial cells of 
the bovine aorta has been mentioned. Sallam et al. Crocin has a protective effect by reducing the production of active oxygen species and decreasing intracellular calcium concentrations (Sallam and Laher 2016). Valko et al. investigated the effect of Crocin on the activity of antioxidant enzymes during an eccentric activity session of inactive men (Valko, et al. 2016). According to the findings of this

study, induction of Crocin before the eccentric activity has a preventive effect on the reduction of oxidative stress following the eccentric activity.

In conclusion, the findings of this study showed that oxidative stress increases cardiomyocyte apoptosis. Also, regular forced swimming, as well as the use of antioxidant agents, Crocin, can have a protective effect on myocardial infarction and cardiomyocyte apoptosis. These effects imply signaling pathways alteration through negative regulation of apoptotic BAX, Caspase 3 expression, and positive regulation of anti-apoptotic gene expression of BCL-2. On the other hand, the combination of forced swimming and Crocin has a synergetic effect on inhibiting apoptotic expression of BAX, caspase 3, and also enhancing the expression of the anti-apoptotic gene of BCL-2 in poisoned rats.

\section{Conflict of interest}

Authors have no conflicts of interest to disclose.

\section{Acknowledgments}

This research work has been extracted from the doctoral dissertation of Mina Akbari, a graduate of Teacher Training Shahid Rajaei University. Thanks to the research assistant and all colleagues.

\section{Funding}

This research was not funded by any organization

\section{Compliance with Ethical Standards}

This article is based on the principles of the Animal Work Ethics Committee. 


\section{References}

1. Arshadi, Sajad, et al.

2015 Evaluation of Trigonella foenum-graecum extract in combination with swimming exercise compared to glibenclamide consumption on type 2 Diabetic rodents. Food \& nutrition research 59(1):29717.

2. Aruoma, Okezie I

1998 Free radicals, oxidative stress, and antioxidants in human health and disease. Journal of the American oil chemists' society 75(2):199-212.

3. Ascensão, António, Rita Ferreira, and José Magalhães

2007 Exercise-induced cardioprotection-biochemical, morphological and functional evidence in whole tissue and isolated mitochondria. International journal of cardiology 117(1):16-30.

4. Faria, Alyssa, and Shanta J Persaud

2017 Cardiac oxidative stress in diabetes: mechanisms and therapeutic potential. Pharmacology \& therapeutics 172:50-62.

5. Ghavami, Saeid, et al.

2005 Apoptosis in liver diseases-detection and therapeutic applications. Medical science monitor 11(11):RA337-RA345.

6. Granger, D Neil, and Peter R Kvietys

2015 Reperfusion injury and reactive oxygen species: the evolution of a concept. Redox biology 6:524-551.

7. Halliwell, Barry, and John MC Gutteridge

2015 Free radicals in biology and medicine: Oxford University Press, USA.

8. Kelly, Frank J

2003 Oxidative stress: its role in air pollution and adverse health effects. Occupational and environmental medicine 60(8):612-616.

9. Lajoie, Claude, Angelino Calderone, and Louise Béliveau

2004 Exercise training enhanced the expression of myocardial proteins related to cell protection in spontaneously hypertensive rats. Pflügers Archiv 449(1):26-32.

10. Maletic, SD, et al.

1999 Alterations of rat reticulocyte (anti) oxidant status and energy metabolism influenced by hydrogen-peroxide. Acta Biologica Iugoslavica. Serija C.

11. MOHSENIZADEH, NAEMEH, et al. 2017 THE SIMULTANEOUS EFFECT OF REGULAR EXERCISE AND VITAMIN D ON NF- $\kappa$ BP65 LEVELS IN MALE RATS EXPOSED TO HYDROGEN PEROXIDE.

12. Moradi, Ali, Seyed Ali Hosseini, and Masoud Nikbakht

2019 Anti-apoptotic Effects of Interval and Continued Training and Crocin on the Muscle Tissue of the Rats with Type II Diabetes Induced by a High-fat Diet. Journal of Nutrition, Fasting and Health 7(3):130-137.

13. Oruc, Serdar, et al.

2016 The antioxidant and antiapoptotic effects of crocin pretreatment on global cerebral ischemia reperfusion injury induced by four vessels occlusion in rats. Life sciences 154:7986. 
14. Persson, Torbjörn, Bogdan O Popescu, and Angel Cedazo-Minguez

2014 Oxidative stress in Alzheimer's disease: why did antioxidant therapy fail? Oxidative medicine and cellular longevity 2014.

15. Pisoschi, Aurelia Magdalena, and Aneta Pop

2015 The role of antioxidants in the chemistry of oxidative stress: A review. European journal of medicinal chemistry 97:55-74.

16. Plant, David R, et al. 2003 Endurance training adaptations modulate the redox-force relationship of rat isolated slow-twitch skeletal muscles. Clinical and experimental pharmacology and physiology 30(1-2):77-81.

17. Quindry, John, et al.

2005 Exercise training provides cardioprotection against ischemia-reperfusion induced apoptosis in young and old animals. Experimental gerontology 40(5):416-425.

18. RADak, ZSOLT, et al.

1999 The effect of exercise training on oxidative damage of lipids, proteins, and DNA in rat skeletal muscle: evidence for beneficial outcomes. Free Radical Biology and Medicine 27(1-2):69-74.

19. Radák, Zsolt, et al.

2000 Exercise preconditioning against hydrogen peroxide-induced oxidative damage in proteins of rat myocardium. Archives of biochemistry and biophysics 376(2):248-251.

20. Roy, Sophie, and Donald W Nicholson

2000 Cross-talk in cell death signaling. The Journal of experimental medicine 192(8):F21-F26.

21. Sallam, Nada, and Ismail Laher

2016 Exercise modulates oxidative stress and inflammation in aging and cardiovascular diseases. Oxidative medicine and cellular longevity 2016.

22. Valko, Marian, et al.

2016 Redox-and non-redox-metal-induced formation of free radicals and their role in human disease. Archives of toxicology 90(1):1-37.

23. Verma, SK, and A Bordia

1998 Antioxidant property of saffron in man. Indian journal of medical sciences 52(5):205-207.

24. Xiang, Min, et al.

2006 Crocetin prevents AGEs-induced vascular endothelial cell apoptosis. Pharmacological research 54(4):268-274.

25. Zhang, Xiaoyan, et al.

2020 Cognitive Protective Mechanism of Crocin Pretreatment in Rat Submitted to Acute High-Altitude Hypoxia Exposure. BioMed Research International 2020. 
Figures

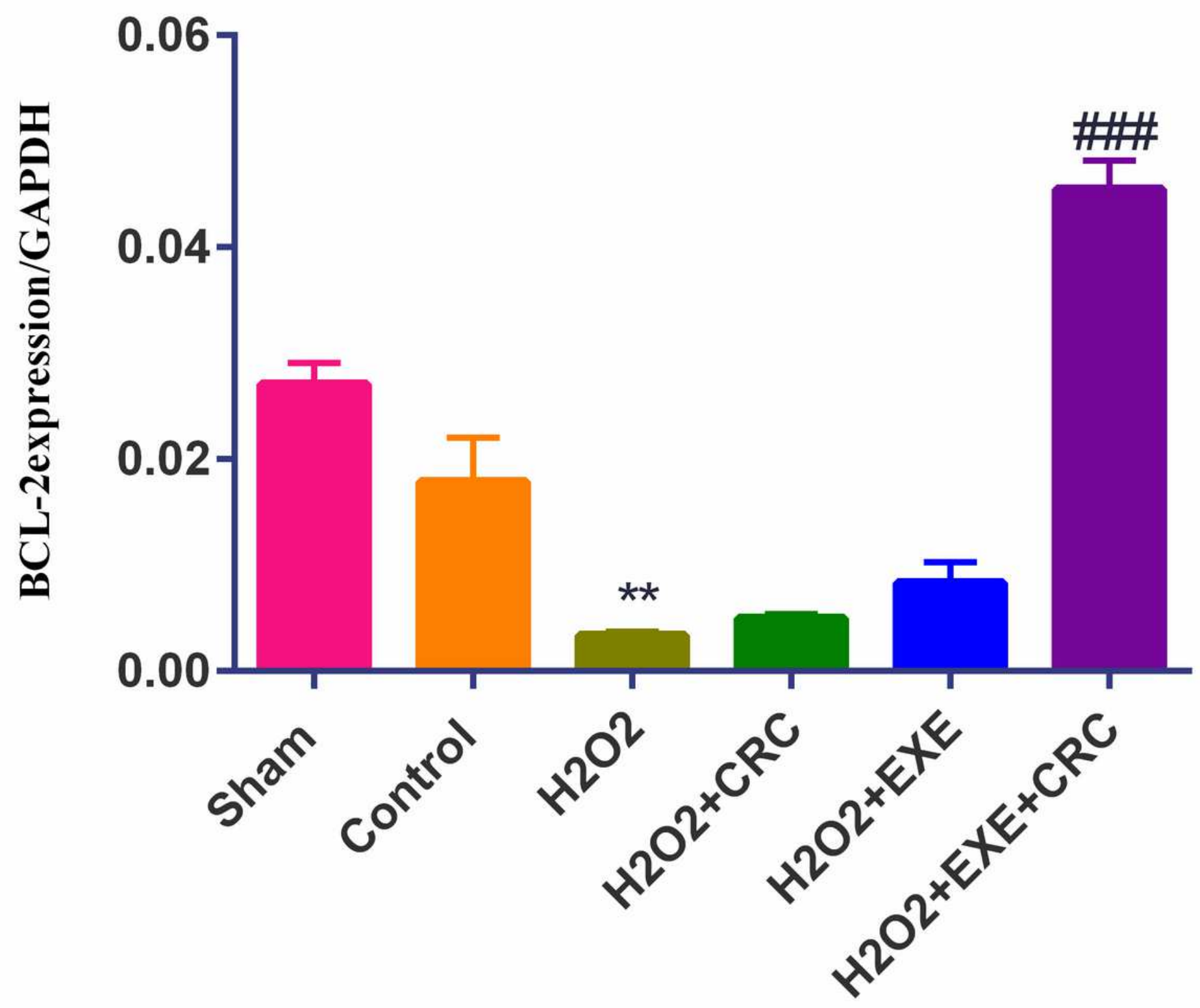

Figure 1

BCL-2 expression 


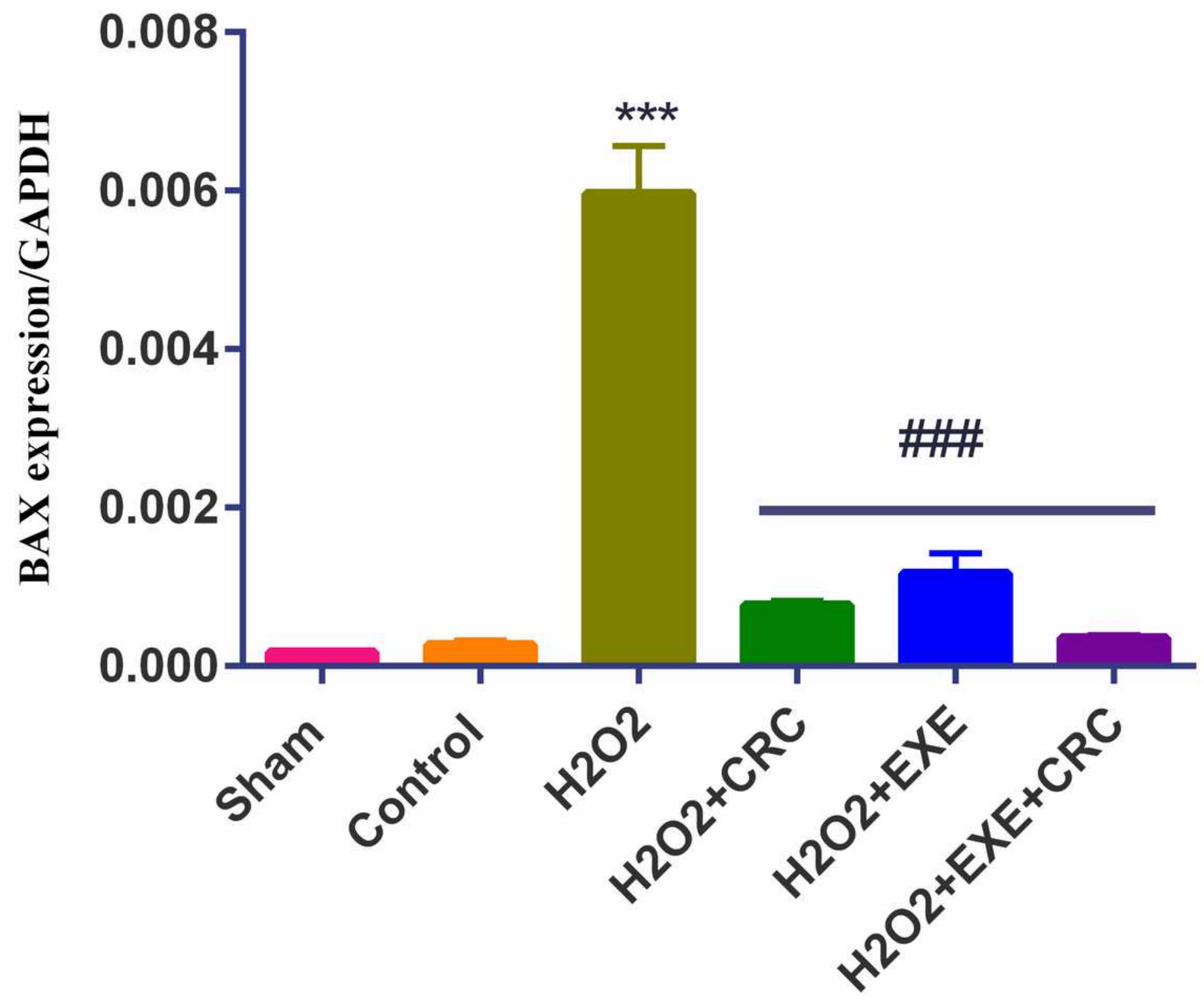

Figure 2

BAX expression 


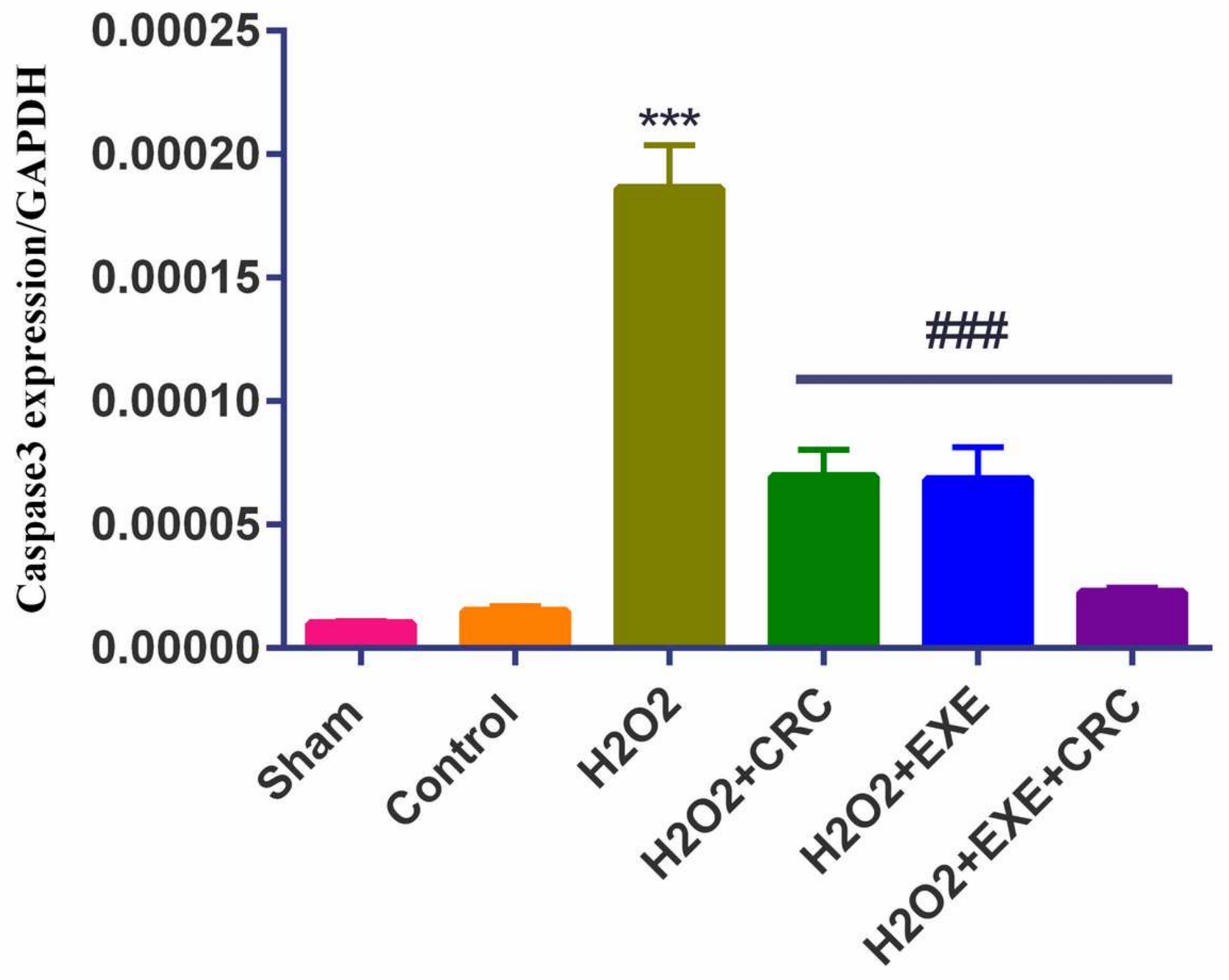

Figure 3

Caspase 3 expression 

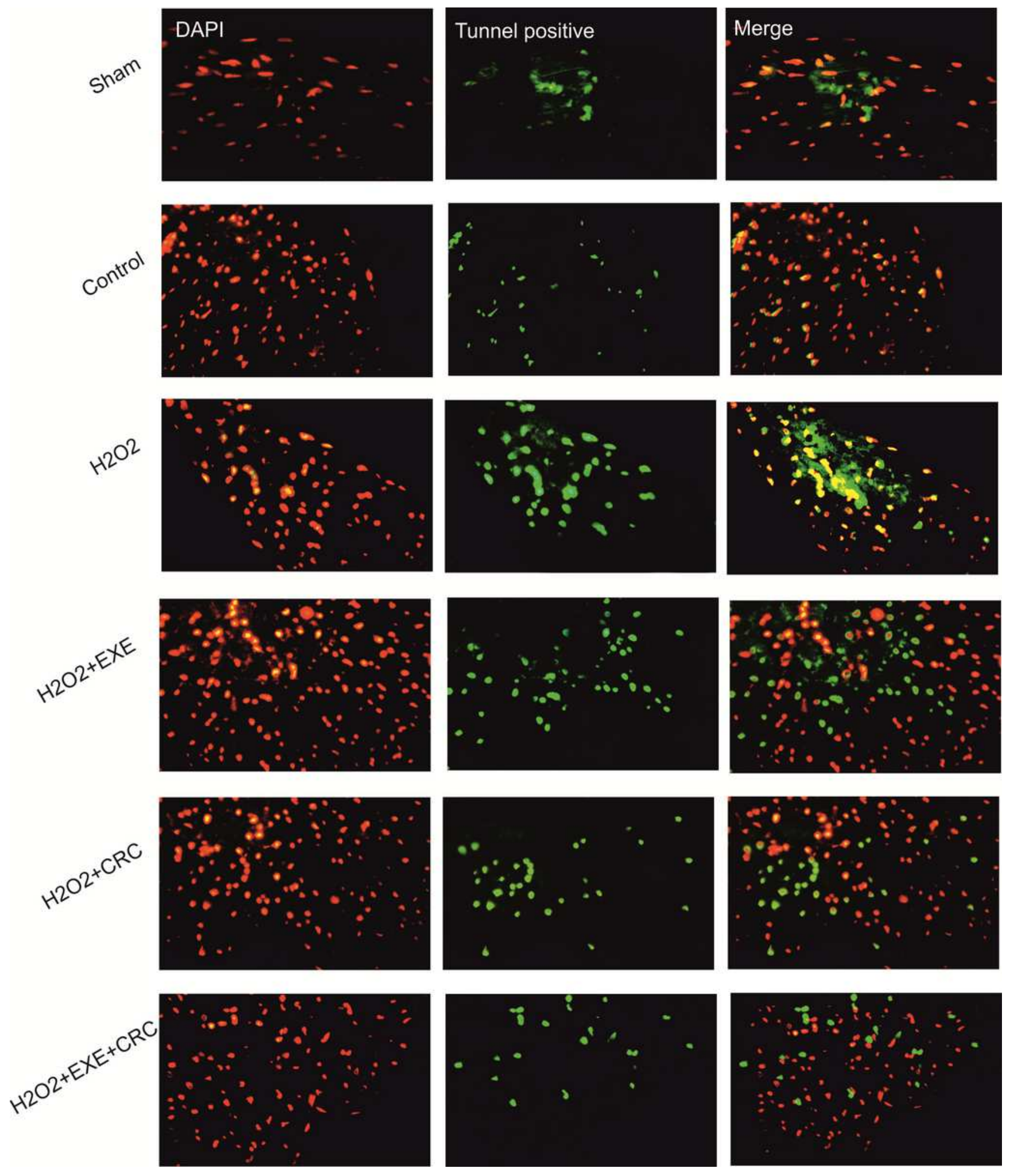

Figure 4

Effect of crocin and exercise on apoptosis

\section{Supplementary Files}

This is a list of supplementary files associated with this preprint. Click to download. 
- Table1.pdf 